\title{
Relação entre Estilos de Aprendizagem e forma de navegação em Apresentações Paralelas Multimídia
}

\author{
Relationship between Learning Styles and Way of \\ Navigation in Parallel Presentations Multimedia
}

\author{
Manuel Constantino Zunguze \\ Universidade Federal do Rio Grande do Sul \\ Felipe Becker Nunes \\ Universidade Federal do Rio Grande do Sul \\ Kelly Hannel \\ Universidade Federal do Rio Grande do Sul \\ Sérgio Roberto Kieling Franco \\ Universidade Federal do Rio Grande do Sul \\ José Valdeni De Lima \\ Universidade Federal do Rio Grande do Sul
}

\begin{abstract}
Resumo: Vários estudos na esfera acadêmica têm sido conduzidos relacionando estilos de aprendizagem de estudantes e aprendizagem adaptativa. Neste trabalho visa verificar-se se a forma de navegação dos estudantes em apresentações paralelas multimídia leva em consideração os estilos de aprendizagem preferenciais dos mesmos e se a relação existente entre os estilos de aprendizagem e a forma de navegação influencia positivamente no aproveitamento pedagógico. Participaram da pesquisa 74 estudantes do curso de Licenciatura em Informática e Engenharia Electrónica da Universidade Pedagógica de Moçambique. Os resultados do estudo mostraram que um número significativo de estudantes interagem com os objetos de aprendizagem considerando os seus estilos de aprendizagem. Foi também possível verificar melhora significativa no desempenho dos estudantes considerando-se a metodologia aplicada.
\end{abstract}

Palavras-chave: Estilos de Aprendizagem. Formas de Navegação. Apresentações Adaptativas Multimídias.

\begin{abstract}
Several studies in the academic sphere have been conducted relating student learning styles and adaptive learning. This paper aims to verify if the way students navigate in parallel multimedia presentations take into account their preferred learning styles and if the relationship between learning styles and the way of navigation positively influences pedagogical achievement. Participated in the survey 74 students of the degree in Computer Science and Electronic Engineering of the Pedagogical University of Mozambique. The results of the study showed that a significant number of students interact with the learning objects considering their learning styles. It was also possible to verify significant improvement in students' performance considering the applied methodology.
\end{abstract}

Keywords: Learning Styles. Ways of Navigation. Adaptive Multimedia Presentations

ZUNGUZE, Manuel Constantino; NUNES, Felipe Becker; HANNEL, Kelly; FRANCO, Sérgio Roberto Kieling; VALDENI, Jusé entre Estilos de Aprendizagem e forma de navegação em Apresentaçôes Paralelas Multimídia. Informática na Educação: teoria \& prática, Porto Alegre, v. 20, n.1, p. 15-26, jan./abr. 2017. 


\section{Introdução}

As tecnologias da informação têm evoluído constantemente nos últimos anos, e os sistemas educacionais não permanecem alheios a essa evolução. Como exemplo, pode-se citar os sistemas adaptativos voltados a educação. Na sua larga maioria, tais sistemas tendem a personalizar o ensino considerando as preferências dos estudantes relacionadas aos estilos de aprendizagem. Estudantes com diferentes estilos de aprendizagem abordam a aprendizagem de forma diferente. Estudos relacionados aos estilos de aprendizagem vêm sendo realizados há bastante tempo como apontam os estudos de Kolb e Felder. Em pesquisas recentes os estilos de aprendizagem são mais utilizados como forma de personalizar o ensino.

O ensino personalizado é um método de instrução que exige que os estudantes sejam abordados individualmente (CAKIR e SIMSEK, 2010). Esse método de ensino encara o estudante como um indivíduo com caraterísticas únicas. A individualização do ensino é conseguida através da instrução centrada no estudante, projetando a instrução de acordo com as necessidades individuais dos estudantes (DIACK, 2004).

A personalização é uma forma de adaptar o conteúdo, neste estudo aborda-se a aprendizagem adaptativa, onde segundo Oxman e Wong (2014), a aprendizagem adaptativa é um dos métodos destinados à aquisição de conhecimentos de forma dinâmica. Ela suporta sistemas educacionais baseados no computador que ajusta a apresentação de conteúdos em resposta ao desempenho do estudante. Basicamente, a aprendizagem adaptativa inclui três elementos fundamentais: o modelo de conteúdo (objetos de aprendizagem), modelo do aprendiz (estudante) e modelo instrucional.

Partindo do princípio que a aprendizagem adaptativa visa personalizar o ensino, uma sequência ou caminhos de aprendizagem podem ser criados e eles variam de estudante para estudante. Essa sequência ou caminhos de aprendizagem denomina-se Trajetórias de Aprendizagem. Segundo Canto Filho et al. (2016), Trajetórias de Aprendizagem são sequências de trajetos, onde trajeto é a apropriação de competências através do processo de ensino e aprendizagem tendo como ponto de partida competências ou conceitos previamente conhecidos. As trajetórias de aprendizagem podem subdividir-se em trajetórias planejadas - conjunto de atividades planejadas com o objetivo de alcançar um determinado objetivo - e, trajetórias realizadas - atividades realizadas pelo aprendiz (estudante) com o objetivo de alcançar um determinado objetivo educacional.

Ocepek et al. (2013) defendem que estudantes tendem a preferir determinadas mídias em detrimento a outras, de acordo com o estilo de aprendizagem. Neste sentido, integrando a aprendizagem adaptativa aos estilos de aprendizagem, cada estudante cria sua própria trajetória de aprendizagem para alcançar objetivos previamente definidos.

Adaptar conteúdos às necessidades do estudante e, desta forma personalizar o ensino, exige um certo conhecimento das preferências ou background educacional do estudante. No contexto do presente estudo procurou-se adaptar objetos de aprendizagem multimídia às necessidades do estudante, levando em consideração os seus estilos de aprendizagem preferenciais.

O presente artigo objetivou estudar a forma de navegação dos estudantes em apresentações paralelas multimídia considerando os estilos de aprendizagem preferenciais dos estudantes e ainda verificar se a relação existente entre os estilos de aprendizagem e a forma de navegação influencia positivamente no aproveitamento pedagógico. Trata-se da continuação do estudo iniciado em Zunguze et al. (2016) e é um estudo de natureza explicativa.

\section{Trabalhos Relacionados}

Ao longo do tempo várias pesquisas vêm sendo conduzidas mostrando que estudantes aprendem de forma diferenciada e que o uso de uma única estratégia instrucional para ensinar todos os estudantes tem um impacto negativo sobre os resultados de aprendizagem pois as maneiras de aprender divergem. A seguir são apresentadas algumas pesquisas que relacionam 
os estilos de aprendizagem de estudantes em diferentes contextos com o objetivo de personalizar o ensino.

Waes et al. (2014) apresentam um estudo e uma metodologia para investigar se estudantes com diferentes estilos de aprendizagem fazem uso do potencial da flexibilidade de materiais de aprendizagem online no contexto de um centro de escrita online. O estudo pretendia investigar o efeito dos estilos de aprendizagem sobre a abordagem do estudante para as tarefas de escrita (processo), e sobre as cartas que eles escreviam (produto). Foi encontrado um efeito dos estilos de aprendizagem: os escritores ativos e reflexivos abordaram a tarefa de forma diferente, mas apenas no início do processo. Na fase inicial, os estudantes reflexivos eram mais propensos a se concentrar na seção de teoria do que os estudantes ativos. Isso sugere que escritores com estilos de aprendizagem diferentes abordam os materiais de aprendizagem de diferentes maneiras, muitas vezes em harmonia com as preferências que caracterizam seus estilos de aprendizagem.

O estudo de Ocepek et al. (2013) é focado em projetar um sistema de aprendizagem relacionando combinações de diferentes estilos de aprendizagem aos tipos preferidos de materiais multimídia. Os autores exploram um modelo de decisão que visa propor materiais de aprendizagem multimídia. O modelo de decisão resultante mostrou que os estudantes preferem textos de aprendizagem bem estruturados com discriminação de cores e que o modelo de estilo de aprendizagem hemisférico é o critério mais importante para decidir as preferências dos alunos para diferentes materiais de aprendizagem multimídia.

O trabalho de Canto et al. (2015) faz uma análise quantitativa comparando estilos de aprendizagem de estudantes que evadem e que persistem na disciplina de Cálculo Diferencial e Integral I do curso de Engenharia. Neste trabalho os autores constataram que não há diferenças nos estilos de aprendizagem dos dois grupos de estudantes.Portanto, para eles, os estilos de aprendizagem preferenciais não influenciam na persistência ou evasão de estudantes do curso de Engenharia na disciplina de Cálculo. As pesquisas apresentadas anteriormente visam investigar a relação existente entre os estilos de aprendizagem e as preferências de materiais de aprendizagem dentro de contextos específicos do processo de ensino e aprendizagem. Elas mostram estar relacionadas ao estudo desenvolvido, onde procura-se verificar a existência de relação entre os estilos de aprendizagem preferenciais dos estudantes e a forma de navegação em um ambiente de aprendizagem adaptativo multimídia composto por vídeo-aulas e objetos de aprendizagem interativos. O diferencial entre a presente pesquisa e as demais reside na forma de apresentação dos objetos de aprendizagem dentro do ambiente. Nesta pesquisa, os objetos encontram-se dispostos de forma paralela. Além disso, foi realizada a integração da aprendizagem adaptativa com os estilos de aprendizagem visando traçar trajetórias de aprendizagem dos estudantes.

\section{Fundamentação Teórica}

Este trabalho tem como objetivo fazer um estudo da forma de navegação dos estudantes em apresentações paralelas multimídia considerando os estilos de aprendizagem preferenciais dos estudantes, para verificar a existência de relação entre os mesmos e se essa relação influencia positivamente no aproveitamento pedagógico. A seguir, são apresentados os principais temas abordados neste estudo.

\subsection{Aprendizagem Adaptativa}

Aprendizagem adaptativa refere-se genericamente a um processo de aprendizagem em que o conteúdo ensinado ou a maneira como o conteúdo é apresentado muda ou "se adapta", com base nas respostas de cada estudante (OXMAN e WONG, 2014). Segundo os autores, a aprendizagem adaptativa ajusta dinamicamente o nível ou tipo de instrução com base nas habilidades ou preferências individuais do estudante, e ajuda a personalizar a instrução para melhorar ou acelerar o desempenho do estudante. 
O termo "adaptativo" está associado a uma gama de diversas características e capacidades de sistemas na indústria de e-learning, tornando assim necessário qualificar as qualidades que se atribuem a um sistema quando se utiliza o termo (PARAMYTHIS e LOIDL-REISINGER, 2004). Os sistemas de aprendizagem adaptativa, na prática, podem variar drasticamente quanto a sofisticação, nível de detalhe, e até mesmo a diversidade de modelos de interface do usuário.

Embora possa haver exceções, os sistemas de aprendizagem adaptativa são geralmente construídos de três elementos principais: modelo do conteúdo, modelo do estudante e modelo de instrução (OXMAN e WONG, 2014). Ainda segundo os autores, o objetivo de um sistema de aprendizagem adaptativa é personalizar a instrução, a fim de melhorar ou acelerar o ganho no desempenho de um estudante. Em sua essência, tais sistemas são utilizados para identificar o que o estudante faz, o que ele não entende, identificar e fornecer conteúdos que irão ajudar o estudante a aprender até que alguma meta de aprendizado definida seja atingida.

Brusilovsky (2001) distingue dois tipos de adaptatividade para sistemas hipermídia: adaptação a nível de conteúdo ou apresentação adaptativa e adaptação a nível de link ou suporte à navegação adaptativa. O objetivo da apresentação adaptativa é adaptar os conteúdos contidos em uma página aos objetivos e conhecimentos do estudante e ainda as informações armazenadas no modelo do estudante.

Em um sistema com apresentações adaptativas, as páginas não são estáticas, elas são construídas e geradas adaptativamente para cada usuário. O objetivo do suporte à navegação adaptativa é assistir o estudante na orientação e navegação no hiperespaço mudando a aparência dos links visíveis. Por exemplo, um sistema hipermídia adaptativo pode agrupar ou parcialmente ocultar os links de uma página para tornar mais fácil escolher para onde prosseguir.

O suporte à navegação adaptativa ao mesmo tempo guia e deixa o estudante escolher o próximo assunto a ser estudado ou o próximo problema a ser resolvido (BRUSILOVSKY, 2003). No escopo deste trabalho, um ambiente de aprendizagem é considerado adaptativo se for capaz de monitorar e interpretar as atividades dos usuários e inferir as preferências agindo sobre o conhecimento disponível para facilitar a dinâmica do processo de ensino e aprendizagem.

\subsection{Estilos de aprendizagem}

Estilos de aprendizagem são maneiras individuais de perceber e sistematizar as experiências às quais todos os indivíduos são expostos desde o nascimento, ou seja, são características particulares de aprendizagem (SILVA e SILVA, 2006). Cada indivíduo possui um estilo único e diferenciado no processo de aquisição de conhecimentos.

No escopo desta pesquisa, foi selecionado o modelo de Felder e Silverman por melhor se adequar aos objetivos propostos no desenvolvimento deste trabalho. No modelo proposto por Felder e Silverman (1988), as dimensões dos estilos de aprendizagem estão relacionadas com a forma de captação, percepção, organização, processamento e compreensão da informação.

Após alguns anos de pesquisa, Felder propõe algumas alterações no seu modelo passando a ter 4 dimensões: percepção da informação (sensorial - intuitivo), retenção da informação (visual - verbal), processamento da informação (ativo - reflexivo) e organização da informação (sequencial - global). Felder e Soloman (1991) desenvolveram o Index of Learning StylesQuestionnaire (ILS), que é um instrumento online que identifica os estilos preferenciais de aprendizagem de estudantes a partir do modelo Felder-Silverman, o qual é utilizado na presente pesquisa.

Considerando a aprendizagem adaptativa e que cada estudante aprende de forma diferenciada dependendo do seu estilo cognitivo ou estilo de aprendizagem, diferentes trajetórias de aprendizagem podem ser traçadas para cada estudante dentro do mesmo conteúdo.

\subsection{Trajetórias de Aprendizagem}

Nos processos de ensino e aprendizagem, os professores são agentes que criam previamente trajetórias de aprendizagem a serem seguidas pelos estudantes, e os estudantes 
seguem com certa flexibilidade as trajetórias disponibilizadas por cada professor como processo de aprendizagem.

Simon (1995), propôs a noção de Trajetória Hipotética de Aprendizagem (THA) e Simon e Tzur (2004) estenderam tal teoria. A THA contém o objetivo de aprendizagem, as atividades de aprendizagem, o pensamento e a aprendizagem em que os estudantes podem se envolver (SIMON, 1995).

Considerando as metas, as atividades e a aprendizagem na qual os estudantes se envolvem compõem-se asTHA's (SIMON, 1995). Para Simon e Tzur (2004), uma THA é uma construção de ensino - a trajetória se refere aos trajetos por onde os estudantes seguem com o objetivo de construir conhecimentos sobre os conteúdos programados pelo professor. Ela é hipotética porque uma trajetória de aprendizagem real não é conhecida com antecedência.

A noção de uma THA implica que o professor tem que imaginar como o pensamento e a aprendizagem em que os estudantes podem se envolver e participar de certas atividades instrucionais, se relacionam com o objetivo de aprendizagem escolhido (GRAVEMEIJER, 2004).Segundo Confrey et al. (2009), trajetória de aprendizagem é uma concepção de pesquisa desenvolvida empiricamente, que descreve uma rede ordenada de construtos desenvolvidos pelo estudante em um processo instrucional, cujo objetivo é através de sucessivos refinamentos de representações, articulações e reflexões, movê-lo de um contexto de ideias informais em direção a um contexto de conceitos progressivamente mais complexos.

As trajetórias de aprendizagem auxiliam o professor no acompanhamento das atividades nos processos de ensino e aprendizagem. Elas dão diretrizes para determinar em que o professor deve se concentrar, compõem a espinha dorsal para o acompanhamento das ações, tanto por parte dos professores como por parte dos estudantes para decisões a serem tomadas visando a melhoria dos referidos processos.

Nesse trabalho, as trajetórias são propostas pelo professor através das mídias paralelas e o estudante tem a liberdade de escolher o tipo de mídia que melhor se encaixa na sua forma de aprender, construindo assim sua trajetória individual.

\section{Metodologia}

O procedimento metodológico que norteou a pesquisa é de natureza explicativa, modalidade quase-experimental e abordagem quantitativa.

Para validar as proposições apresentadas nesta pesquisa, foi realizado um estudo, que se constitui da criação de um sistema voltado ao ensino e aprendizagem denominado "Apresentações Adaptativas Multimídias e Multimodais" (AAMM) em HTML, PHP e JavaScript. A Figura 1 mostra a tela inicial do sistema.

O sistema desenvolvido é voltado para a apresentação de conteúdos de forma paralela, e tem como finalidade explorar a forma que os estudantes interagem com os objetos de aprendizagem multimídia dentro do ambiente de acordo com os seus estilos de aprendizagem. O sistema AAMM apresenta dois frames, em que um dos frames apresentou um Objeto de Aprendizagem (OA) interativo e o outro frame mostrou uma vídeo-aula, conforme Figura 2. 


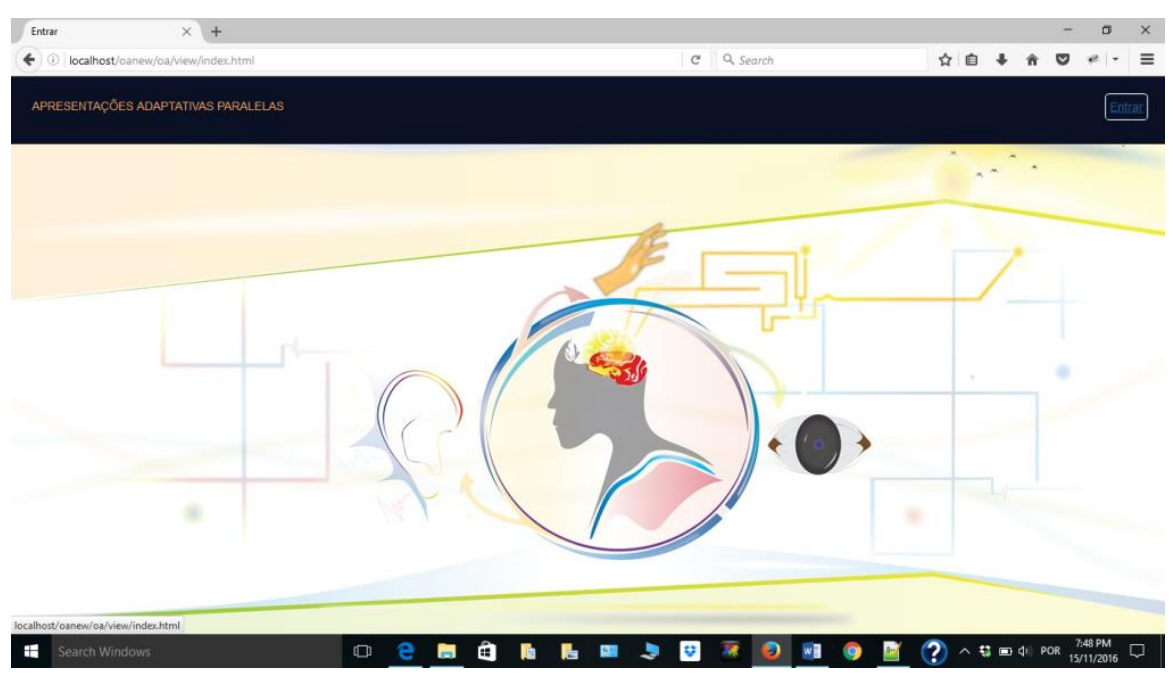

Figura 1 - Login do Sistema AAMM

Fonte: Autor

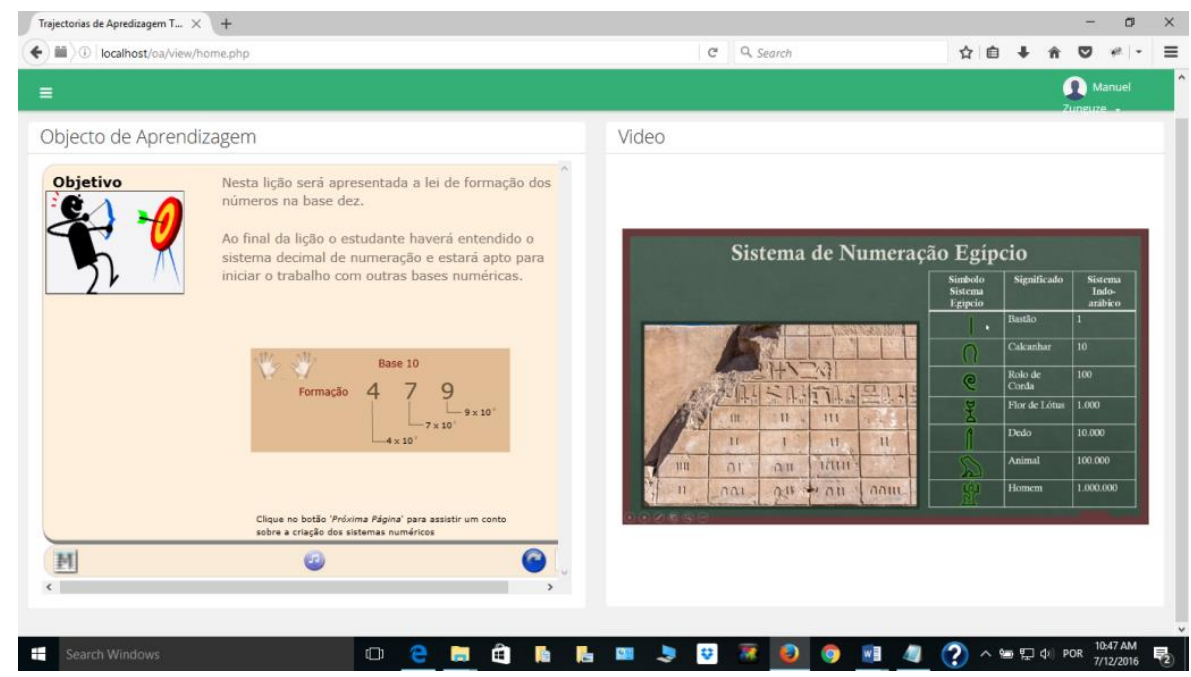

Figura 2 - Disposição dos OAs no sistema AAMM

Fonte: Autor

Os estudantes estavam livres para explorar os OA disponibilizados de acordo com suas preferências, podendo assistir a vídeo-aula, explorar o OA, assistir a vídeo-aula e em seguida explorar o OA ou vice-versa, além de poder explorar o OA e assistir a vídeo aula em simultâneo. Participaram da pesquisa estudantes do curso de Engenharia Eletrônica e Engenharia Informática na disciplina Sistemas de Computação durante oprimeiro semestre de 2016, num total de 74 estudantes.

A seguir são descritos os procedimentos para a coleta de dados:

1. Questionário ILS ${ }^{1}$ para avaliar os estilos de aprendizagem preferenciais dos estudantes. O questionário é composto por 44 questões, com duas alternativas;

${ }^{1}$ O questionário ILS (Index of Learning StylesQuestionnaire) encontra-se disponível em inglês e online através do link https://www.engr.ncsu.edu/learningstyles/ilsweb.html. 
2. Pré-teste e pós-teste, ambos compostos por 20 questões de escolha múltipla. Cada um sobre sistemas de numeração com o objetivo de avaliar os conhecimentos prévios dos estudantes no caso do pré-teste e avaliar a compreensão dos conteúdos ao final da atividade para o caso do pós-teste.As questões do pré-teste eram diferentes das questões do pós-teste, contudo estas questões avaliavam os mesmos conceitos. Tanto o pré-teste quanto o pós-teste, a priori, passaram por uma validação com um grupo

de estudantes diferente dos estudantes participantes da pesquisa.

Foi incluído dentro do ambiente um script capaz de capturar as formas de navegação dos estudantes e armazenar esses dados em uma base de dados para posterior análise e interpretação.

\section{Experimento}

O experimento em questão visava fazer um estudo da forma de navegação dos estudantes em apresentações paralelas multimídia considerando os estilos de aprendizagem preferenciais dos estudantes para verificar a existência de relação entre os mesmos e se essa relação influencia positivamente no aproveitamento pedagógico. Para a efetivação do experimento foi desenvolvido um ambiente de aprendizagem capaz de gerenciar objetos de aprendizagem já existentes.

Os objetos de aprendizagem no ambiente estavam dispostos de formaque possibilitava ao estudante explorá-los de modos imultâneo ou sequencial, conforme a preferência do estudante. Os objetos selecionados para o experimento foram um objeto de aprendizagem interativo desenvolvido em Silverlight ${ }^{2}$ e uma vídeo-aula, ambos sobre o mesmo tópico (Sistemas de Numeração).

O experimento teve duração de 6 semanas. O primeiro encontro foi reservado a explicar aos estudantes sobre as atividades a realizar, cadastramento dos utilizadores no sistema e orientações para responder ao questionário sobre estilos de aprendizagem. No segundo encontro os estudantes foram submetidos ao pré-teste. Após realizar o pré-teste, foram reservadas duas semanas para que os estudantes explorassem os objetos contidos no sistema. Os estudantes foram orientados sobre os procedimentos para realizar as atividades online, interagindo com o ambiente. Esta atividade teve a duração de duas semanas, correspondente a dois encontros presenciais. Posteriormente ao período da interação com o ambiente, os estudantes foram submetidos ao pós-teste.

Como ponto de partida, fez-se a comparação entre as notas do pré-teste e do pós-teste como forma de verificar a evolução entre os dois testes e se o crescimento foi significativo. Para tal foi utilizado o teste estatístico não paramétrico de Wilcoxon devido a distribuição não normal dos dados. A Tabela 1 faz a comparação entre as notas do pré-teste e do pós-teste.

Tabela 1 -Comparação entre notas do pré-teste e pós-teste

\begin{tabular}{|l|l|r|r|r|r|r|l|}
\hline Grupos & N & \multicolumn{1}{|c|}{ Mínimo } & \multicolumn{1}{|c|}{ Máximo } & \multicolumn{1}{l|}{ Mediana } & \multicolumn{1}{l|}{ Média } & Desvio-padrão & p-valor \\
\hline Pré-teste & 74 & 2.0 & 7.0 & 4.5 & 4.4 & 1.2400 & $<0.001$ \\
Pós-teste & 74 & 2.5 & 8.0 & 6.0 & 5.8 & 1.2471 & \\
\hline
\end{tabular}

Fonte: Autor

A partir do $p$-valor da tabela 1 ( $p$-valor igual a 0.001 ), conclui-se que houve um aumento significativo entre as notas do pré-teste e do pós-teste para o grupo dos estudantes que participaram do experimento.

Na Tabela 2 estão apresentados os resultados (percentuais) do questionário ILS dos estilos de aprendizagem preferenciais dos estudantes participantes do estudo por cada dimensão.

\footnotetext{
2 Silverlight é uma ferramenta de desenvolvimento para criar experiências envolventes e interativas para o usuário em aplicações Web e móveis. É um plug-in gratuito, alimentado pela estrutura .NET.
} 
Tabela 2. Estilos de Aprendizagem de estudantes

\begin{tabular}{|c|c|c|c|c|c|c|c|}
\hline \multicolumn{8}{|c|}{ Dimensões dos Estilos de Aprendizagem } \\
\hline \multicolumn{2}{|c|}{ Percepção } & \multicolumn{2}{|c|}{ Retença } & \multicolumn{2}{c|}{ Processamento } & \multicolumn{2}{c|}{ Organização } \\
\hline Sen. & Int. & Vis. & Ver. & Ati. & Ref. & Seq. & Glo. \\
\hline 82.43 & 17.57 & 66.22 & 33.78 & 45.95 & 54.05 & 50.0 & 50.0 \\
\hline
\end{tabular}

Fonte: Autor

Os dados da Tabela 2 mostram a existência de um equilíbrio nas dimensões processamento e organização da informação. Este resultado foi verificado no estudo de Zunguze (2016). Durante o estudo houve necessidade de verificar se efetivamente o estilo de aprendizagem preferencial do estudante, analisando se as respostas do questionário ILS, correspondiam a forma como o estudante explorava os objetos disponibilizados no sistema.

A Figura 3, a seguir, ilustra os cruzamentos entre as respostas do questionário ILS para as dimensões organização e processamento de informação dos estilos de aprendizagem e as formas como os estudantes exploraram efetivamente os objetos disponibilizados no sistema. É importante salientar que os estudantes estavam livres para escolher a forma de navegação entre os objetos disponibilizados no ambiente.

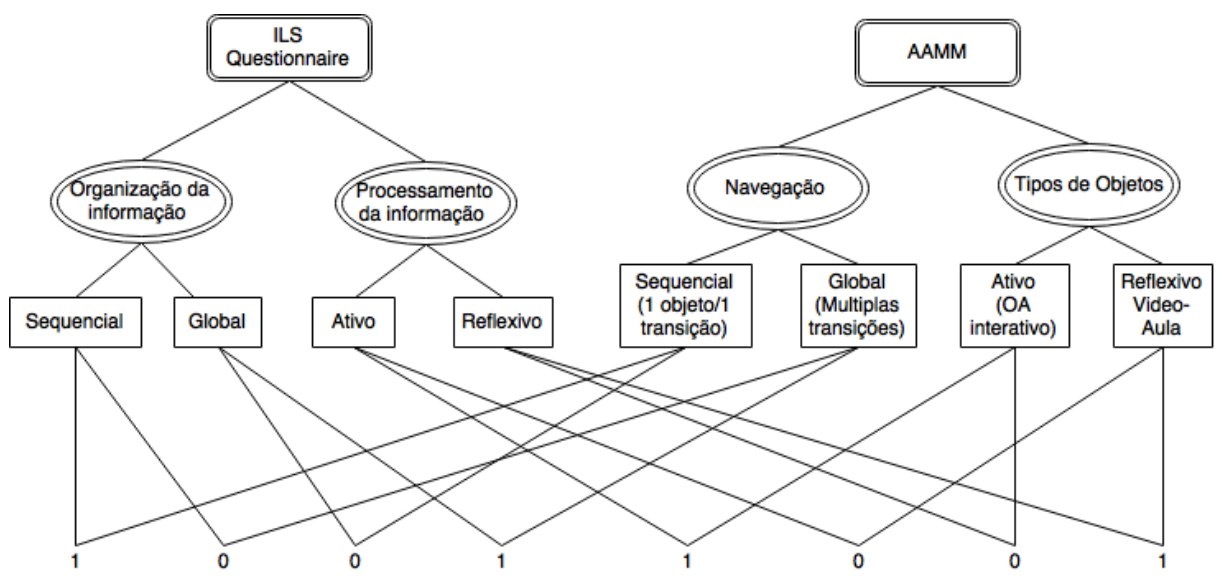

Figura 3 -Cruzamentos entre respostas do Questionário ILS e interações dos estudantes no sistema AAMM

Fonte: Autor

Os valores 0 na Figura 3 significam que em determinada dimensão do EA não houve correspondência entre o resultado do questionário ILS e a forma de navegação do estudante no sistema. Já os valores 1 significam dizer o resultado do questionário ILS correspondia a forma de navegação do estudante no sistema. Na Tabela 3 estão representados os subgrupos dos estudantes distribuídos em termo dos que não houve correspondência total, os que houve correspondência parcial e os que houve correspondência total entre as respostas do questionário ILS e as formas de navegação dos estudantes. 
Tabela 3. Distribuição dos estudantes por dimensão de EA e relação com forma de navegação

\begin{tabular}{|c|c|c|c|c|}
\hline & & \multicolumn{2}{|c|}{ Organização } & \multirow[b]{2}{*}{ Total } \\
\hline & & $\mathbf{0}$ & 1 & \\
\hline \multirow[b]{2}{*}{ Processamento } & $\mathbf{0}$ & 10 & 20 & 30 \\
\hline & 1 & 15 & 29 & 44 \\
\hline \multicolumn{2}{|l|}{ Total } & 25 & 49 & 74 \\
\hline
\end{tabular}

Fonte: Autor

Onúmero 10 na Tabela 3 corresponde ao número de estudantes que o resultado do questionário ILS não correspondeu a forma de navegação no sistema nas duas dimensões. O número 20 corresponde ao número de estudantes em que houve correspondência apenas na dimensão organização da informação. O número 15 corresponde ao número de estudantes em que houve correspondência apenas na dimensão processamento da informação e o número 29 corresponde ao número de estudantes em que houve correspondência entre o EA preferencial e a forma de navegação no sistema.

A partir dos cruzamentos da Figura 2, foi feita uma análise estatística relacionando as dimensões dos EA, as notas do pré-teste e pós-testedos estudantes. Foi utilizado o teste de Wilcoxon. O resultado pode ser observado na Tabela 4 a seguir.

Tabela 4.Teste de Wilcoxon para medianas do pré-teste e pós-teste nas dimensões Organização e Processamento da informação

\begin{tabular}{|l|c|c|c|c|}
\hline & \multicolumn{4}{|c|}{ Dimensão de EA } \\
\hline \multirow{2}{*}{ Medianas } & \multicolumn{2}{|c|}{ Organização } & \multicolumn{2}{c|}{ Processamento } \\
\cline { 2 - 5 } & 0 & 1 & 0 & 1 \\
\hline Pré-teste & 4 & 5 & 4.5 & 4.75 \\
\hline Pós-teste & 5.5 & 6 & 6 & 6 \\
\hline p-valor & 0.005937 & $<0.001$ & $<0.001$ & $<0.001$ \\
\hline
\end{tabular}

Fonte: Autor

O resultado do teste de Wilcoxon mostrou que houve um aumento significativo entre as medianas do pré-teste e do pós-teste ( $p$-valores abaixo de 0.05) em todas dimensões de EA.

A partir dos subgrupos da Tabela 3, foi feita uma análise estatística relacionando as dimensões dos EA, as notas do pré-teste e pós-teste. A Tabela 5 apresenta o tteste de Wilcoxon para os subgrupos dos estudantes das combinações de dimensões.

Tabela 5. Teste de Wilcoxon para os subgrupos dos estudantes das combinações de dimensões

\begin{tabular}{|l|c|c|c|c|}
\hline \multirow{2}{*}{ Medianas } & \multicolumn{4}{|c|}{ Organização - Processamento } \\
\cline { 2 - 5 } & $0-0$ & $1-0$ & $0-1$ & $1-1$ \\
\hline Pré-teste & 3.5 & 5 & 4.5 & 5 \\
\hline Pós-teste & 5.75 & 6 & 5 & 6 \\
\hline p-valor & 0.02195 & 0.00276 & 0.128 & $<0.001$ \\
\hline
\end{tabular}

Fonte: Autor

O teste de Wilcoxon mostrou que houve um aumento significativo entre as medianas do pré-teste e do pós-teste ( $p$-valores abaixo de 0.05) em todos subgrupos nas dimensões de EA, exceto o subgrupo onde houve correspondência apenas na dimensão processamento da 
informação entre as respostas do questionário ILS e a forma de navegação dos estudantes ( $p$ valor igual a 0.128).

O estudo conduzido visava verificar a relação existente entre o estilo de aprendizagem e as formas de navegaçãodos estudantes nos objetos de aprendizagem e se essa relação influenciava positivamente no aproveitamento pedagógico. Analisando os dados da Tabela 5 econsiderando o número de estudantes que compõem o subgrupo pode-se concluir que existe uma relação entre os estilos de aprendizagem e as formas de navegação dos estudantes em objetos de aprendizagem multimídias e multimodais, conforme apresentado na Figura 4.

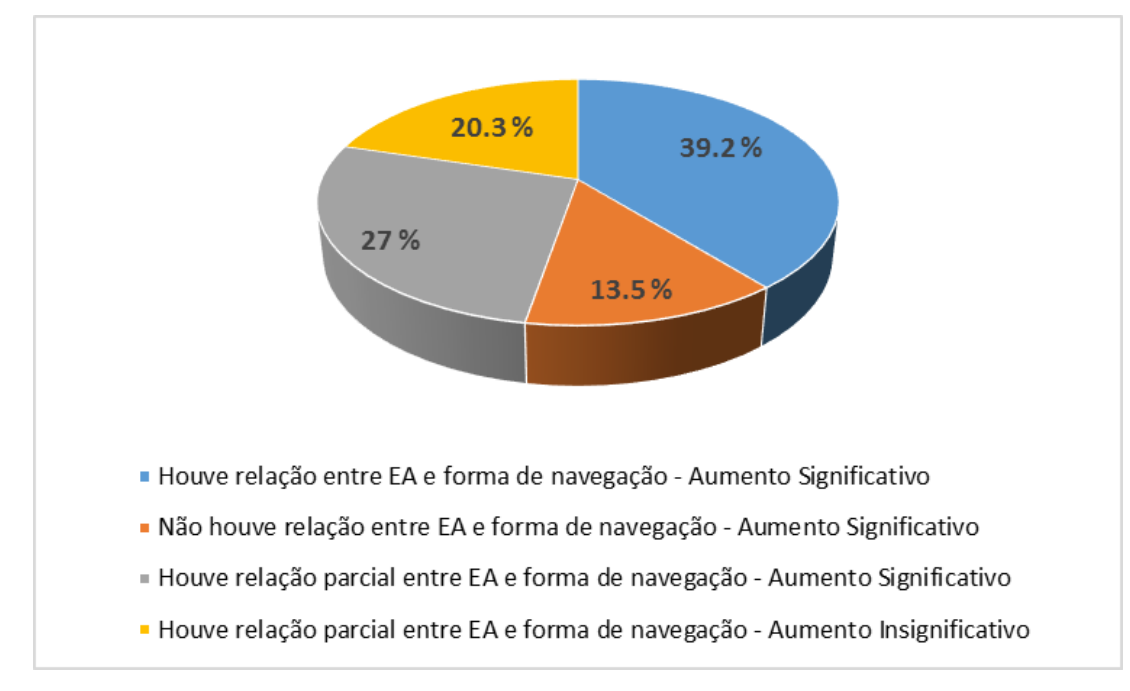

Figura 4. Relação entre EA, forma de navegação e significância do resultado final

Fonte: Autor

Para apenas $13.5 \%$ dos estudantes participantes da pesquisa não houve relação entre os EA e a forma de navegação, embora estes estudantes tenham obtido um crescimento significativo do aproveitamento entre o pré-teste e o pós-teste. Para $39.2 \%$ dos estudantes houve relação entre os EA e a forma de navegação e houve um crescimento significativo do aproveitamento. Já para $27 \%$ dos estudantes houve relação parcial entre os EA e a forma de navegação com um crescimento significativo de aproveitamento. Para os $20.3 \%$ restante dos estudantes houve relação parcial entre os EA e a forma de navegação, mas não houve um crescimento significativo do aproveitamento.

\section{Considerações Finais}

Esse artigo descreve um estudo realizado com estudantes de graduação que teve como objetivo investigar a relação existente entre os EA e a forma de navegação de estudantes em objetos de aprendizagem multimídia e multimodais, verificando se essa relação contribui para a melhoria do aproveitamento do estudante no processo de ensino aprendizagem. Espera-se que o estudo possa contribuir com evidências empíricas para outros estudos relacionados aos EA assim como para estudos relacionados a formas de navegação e interação com objetos de aprendizagem multimídia e multimodais.

Nos últimos tempos estudos que relacionam estilos de aprendizagem e personalização do ensino têm gerado controvérsia no mundo acadêmico. Como exemplo, aponta-se o estudo de Canto et al. (2015) que conclui que a personalização do ensino levando em conta apenas os estilos de aprendizagem dificilmente será eficaz. Por outro lado, a pesquisa de Soflano et al. (2015) mostrou que embora os estilos de aprendizagem dos estudantes identificados numa 
intervenção inicial com o conteúdo de aprendizagem fosse o mesmo que o estilo de aprendizagem identificado através do questionário de estilo de aprendizagem, o estilo de aprendizagem dos estudantes flutuaquando os estudantes interagem com um jogo educativo.

Os resultados desse estudo mostraram que um número significativo de estudantes participantes da pesquisa interage com os objetos de aprendizagem de acordo com o seu próprio estilo de aprendizagem e que isso influencia positivamente para um bom aproveitamento no final do processo de ensino e aprendizagem. Foi também possível verificar uma melhoria significativa no desempenho dos estudantes comparando as notas do pré-teste e do pós-teste.

A partir dos dados coletados no estudo é possível traçar trajetórias de aprendizagem dos estudantes tendo como finalidade personalizar o ensino baseando-se nos estilos de aprendizagem. Portanto, embora o estudo não seja conclusivo, ele pode servir de base para aprofundamento e ou consolidação de futuras pesquisas.

Como trabalhos futuros, este estudo pode se beneficiar de um aumento significativo do número de estudantes participantes, assim como pela relação entre os estilos de aprendizagem e o tipo de objetos de aprendizagem utilizados no experimento (vídeo-aula e objeto de aprendizagem interativo). Neste caso, o estudo estaria relacionado a escolha do objeto de aprendizagem pela sua interatividade tendo em conta o EA preferencial do estudante.

\section{Refêrencias}

ALMEIDA, M. E. B. Educação a distância na internet: abordagens e contribuições dos ambientes digitais de aprendizagem, São Paulo, 2003.

ALVES, L. Educação a distância: conceitos e história no Brasil e no mundo. Revista Brasileira de Aprendizagem e a Distância, v. 10, n. 21, p. 83-92, 2011.

BEChARA, J. J. B.; HAGUENAUER, C. J. Por Uma Aprendizagem Adaptativa Baseada na Plataforma Moodle. Revista EducaOnline. ISSN 1983-2664. Volume 4- no 1- Janeiro/Abril de 2010.

BRUSILOVSKY, P. "Adaptive hypermedia", User Modeling and User Adapted Interaction, p. 87-110, 2001.

BRUSILOVSKY, P. Developing Adaptive Education Hypermedia Systems: From Design Models to Authoring Tools. In Murray, T., Blessing S., \&Ainsworth, S. (eds.), 2003.

CAKIR, O.; SIMSEK, N. A comparative analysis of the effects of computer and paper-based personalizationon student achievement. Computers \& Education, 55(4), pp. 1524-1531, 2010.

CANTO, A. B. DO; MÜLLER, T.; LIMA, J. V. DE. Estilos de Aprendizagem: vale a pena investir? RENOTE, v. 13, n. 1, Julho, 2015.

CANTO FILHO, A. B. ;NUNES, F. B. ; ZUNGUZE, M. C. ; HANNEL, K.; WAGNER, R. ; SIMBINE, F. B. ; LIMA, J. V. . Trajetórias de Aprendizagem. In: José Valdeni de Lima; Manuel Constantino Zunguze; Kelly Hannel; Felipe Becker Nunes; (Org.). Trajetórias de Aprendizagem: teoria e pratica. 1ed.Seattle: Amazon, v. 1, p. $1-10,2016$.

CONFREY, J.; MALONEY, A. P.; NGUYEN, K. H.; MOJICA, G.; MYERS, M. Equipartitioning/splitting as a foundation of rational number reasoning using learning trajectories.Thessaloniki, Greece, 2009.

DIACK, A. Innovation and personalized learning. EducationReview, 18(1), pp. 49-55, 2004.

CaFELDER, R. M; SILVERMAN, L. K. Learning and teaching styles in engineering education. Engineering Education, v. 78, n. 7, p. 674-681, 1988.

FELDER, R. M; SOLOMAN, B. A. Learning styles and strategies. 1991. Disponível em: http://www4.ncsu.edu/unity/lockers/users/f/felder/public/ILSdir/styles.htm. Acesso em 21 abril 2015.

GRAVEMEIJER, K. Creating opportunities for students to reinvent mathematics. Presented at the ICME 10, Denmark, 2004.

OCEPEK, U., BOSNIĆ, Z., ŠERBEC, I. N., andRUGELJ, J. Exploring the relation between learning style models and preferred multimedia types. Computers \& Education, 69:343-355. 2013.

OXMAN, S.; WONG, W. Adaptive Learning Systems. DeVry Education Group and Integrated Education Solutions. 2014 
PARAMYTHIS, A.; LOIDL-REISINGER, S. Adaptive Learning Environmentsand e-Learning Standards. Electronic Journal on e-Learning Volume 2, 181-194, 2004

SILVA, W. M.; SILVA, E. C. L. Investigação dos Dados sobre Estilos de Aprendizagem dos Alunos frequentadores da base de apoio ao Aprendizado Autônomo. Revista Científica da UFPA, 2006.

SIMON, M. Reconstructing mathematics pedagogy from a constructivist perspective. Journal for Research in Mathematics Education, p.114-145, 1995.

SIMON, M.; TZUR, R. Explicatingthe role of mathematical tasks in conceptual learning: An elaboration of the hypothetical learning trajectory. Mathematical Thinking and Learning, 6(2), p. 91-104, 2004.

SOFLANO, M.; CONNOLLY, T.M.;HAINEY, T. Learning styleanalysis in adaptive GBL applicationtoteach SQL. Computers \& Education, 86, pp. 105-119, 2015

WAES, L. V.; WEIJEN, D. V.; LEIJTEN, M. Learning towrite in an online writing center: The effect of learning styles on the writing process. Computers \& Education, Volume 73, p. 60-71, 2014.

ZUNGUZE, M. C.; NUNES, F. B.; LIMA, J. V.; FRANCO, S. R. K. Adaptatividade de Apresentações Paralelas Multimídia: Trajetórias de Aprendizagem Temporais. In: XXIV Ciclo de Palestras Sobre Novas Tecnologias na Educação, 2016, Porto Alegre. Anais do XXIV Ciclo de Palestras Sobre Novas Tecnologias na Educação, v. 1. p. 21-30, 2016.

Submetido para avaliação em 15 de dezembro de 2016 Aprovado para publicação em 22 de março de 2017

\section{Manuel Constantino Zunguze}

Programa de Pós-Graduação em Informática na Educação - Universidade Federal do Rio Grande do Sul UFRGS, Brasil, manuelczunguze@gmail.com

\section{Felipe Becker Nunes}

Programa de Pós-Graduação em Informática na Educação - Universidade Federal do Rio Grande do Sul UFRGS, Brasil, nunesfb@gmail.com

\section{Kelly Hannel}

Programa de Pós-Graduação em Informática na Educação - Universidade Federal do Rio Grande do Sul UFRGS, Brasil, khannel@gmail.com

Sérgio Roberto Kieling Franco

Programa de Pós-Graduação em Informática na Educação - Universidade Federal do Rio Grande do Sul UFRGS, Brasil, sergio.franco@ufrgs.br

José Valdeni de Lima

Programa de Pós-Graduação em Informática da Educação e Programa de Pós-Graduação em Ciência da Computação - Universidade Federal do Rio Grande do Sul - UFRGS, Brasil, valdeni@inf.ufrgs.br 